\title{
BRECHA DE INGRESOS LABORALES POR GENERO EN BOLIVIA. UN ANALISIS DE SU EVOLUCION EN EL PERIODO 1993 A 2018*
}

THE GENDER LABOR INCOME GAP IN BOLIVIA. AN ANALYSIS OF ITS EVOLUTION BETWEEN 1993 AND 2018

\section{MANUEL URQUIDI** \\ Banco Interamericano de Desarrollo}

\section{HORACIO VALENCIA***}

CAF-Banco de Desarrollo de América Latina

\section{GUILLAUME DURAND****}

Banco Interamericano de Desarrollo

\section{Resumen}

En Bolivia las mujeres reciben menores remuneraciones que los hombres en trabajos similares, además de estar concentradas en trabajos de baja remuneración. El presente documento analiza la evolución de la brecha de ingresos por género en el periodo entre 1993 a 2018 en Bolivia. Utilizando encuestas de hogares y dos modelos de medición de las diferencias en el ingreso laboral, la descomposición Blinder-Oaxaca y la descomposición Nopo, se encuentra evidencia de una importante reducción en la brecha de ingreso por género en el periodo observado. Esta reducción se explica tanto por la reducción de diferencias en las características observables que afectan el mercado laboral, principalmente educación y el impacto de las características familiares respecto del acceso al empleo. El segundo componente que explica la reducción de la brecha se debe a la reducción

\footnotetext{
* Las opiniones expresadas en esta publicación son de exclusiva responsabilidad de los autores. Se agradecen los valiosos comentarios de Nelson Chacón, Alexandre Bagolle y Alejandro Quevedo. Agradecemos especialmente la revisión y comentarios de Claudia Piras.

** E-mail: manuelu@iadb.org

*** E-mail: horaciovalenciar@gmail.com

**** E-mail: guillaumedurand17@gmail.com
} 
del componente no explicado de la brecha, que se asume generalmente como discriminación.

Palabras clave: Economía de género, diferencias salariales, discriminación.

Clasificación JEL: J16, J31, J71.

\begin{abstract}
In Bolivia, women working in similar positions as men face lower remunerations, besides being concentrated in low-paid jobs. This paper sheds lights over the gender labor income gap evolution in Bolivia over a 25-year period (1993 to 2018). Using household surveys and applying two different methods, the Blinder-Oaxaca decomposition and the Nopo decomposition, we find sound evidence of an important reduction in the gender labor income gap during the analyzed period. This reduction is due to, firstly, the reduction of the number of observable characteristics affecting the labor market such as education and the impact of family characteristics on access to employment. Secondly, it shows the reduction in the unexplained component, which is usually assumed to be discrimination.
\end{abstract}

Keywords: Gender economics, wage differentials, discrimination.

JEL Classification: $J 16, J 31, J 71$.

\title{
1. INTRODUCCION
}

América Latina y el Caribe (ALC) han mostrado grandes avances hacia la igualdad de género (Chioda, 2011). La visibilidad de las mujeres en el hogar, en la escuela, en el mercado laboral y en el ámbito político ha evolucionado significativamente en estos últimos años (Ñopo, 2012). Sin embargo, y a pesar de todos estos avances, en la mayoría de los países de ALC los hombres ganan más que las mujeres por un mismo trabajo y las diferencias en el ingreso laboral en favor de los hombres persisten. Constituyéndose todavía como una de las formas más injustificables de desigualdad (OIT, 2019).

En Bolivia, a pesar de los avances normativos en temas relacionados a la equidad de género, las mujeres aún sufren de discriminación en diferentes ámbitos. Además del alto nivel de violencia doméstica y machismo, las mujeres presentan menores oportunidades de estudio, asistencia médica, oportunidades laborales, perciben menores ingresos laborales y presentan una mayor probabilidad de ser pobres. 
En lo que se refiere al ámbito económico, Bolivia se encuentra en el puesto 105 (de 153) en el ámbito de participación y oportunidades económicas dentro del Índice Global de Brechas de Género del Foro Económico Mundial (WEF, 2020), donde destaca particularmente la inequidad en los ingresos entre hombres y mujeres ante trabajos similares, ocupando el puesto 134 de 153. Asimismo, datos del Sistema de Información de Mercados Laborales y Seguridad Social del Banco Interamericano de Desarrollo (BID) confirman este hecho y muestran que en 2018 las mujeres ganaban en promedio $82 \%$ de lo que gana su contraparte masculina por hora trabajada, siendo que el promedio de la región era de $93^{1}$. Adicionalmente, alrededor de $50 \%$ de las mujeres presenta ingresos laborales mensuales menores al salario mínimo nacional y tienen una mayor probabilidad a tener peores condiciones laborales, a desempeñarse en trabajos informales o estar desempleadas.

A pesar de la situación, la disponibilidad de información acerca del tema es todavía limitada y existen solo algunos estudios que analizan la diferencia de ingresos laborales entre mujeres y hombres en Bolivia. Siendo que, además, estos estudios utilizan métodos empíricos diferentes, lo que dificultan la comparabilidad entre los mismos y, por esta razón, el seguimiento de la evolución de la problemática.

El presente documento busca reducir la brecha de conocimiento de la temática al proponer un análisis riguroso de la evolución de la brecha de ingresos entre hombres y mujeres en Bolivia en el periodo 1993 a 2018. Para esto, el documento analiza la evolución de la brecha de ingresos laborales utilizando dos metodologías: primero usa la descomposición de Blinder-Oaxaca y posteriormente la descomposición Nopo, posibilitando además la comparación de evidencia obtenida mediante un modelo paramétrico con un modelo no paramétrico para un periodo amplio de tiempo. La posibilidad de utilizar ambas metodologías nos permite contar con un amplio espectro de análisis y tratar de identificar las variables que estarían determinando su evolución.

Los resultados muestran una importante reducción en la brecha de ingresos laborales por género en los 25 años analizados. Esta reducción puede ser explicada por dos hechos. Primero, por una reducción en las diferencias observables de las mujeres con relación a los hombres, principalmente gracias a la reducción de la brecha educacional de las mujeres en el mercado de trabajo y a cambios en el entorno social y familiar que permitió el ingreso de mujeres al mercado laboral. La segunda parte de la reducción de la brecha de ingresos puede ser entendida por la reducción del componente asociado a la discriminación por género. Adicionalmente, el presente documento encuentra evidencia de que la brecha de ingresos laborales por género es menor a mayores niveles de educación y en el sector formal.

Lo que resta del documento se divide de la siguiente manera: la sección 2 presenta una revisión de la literatura relevante; la sección 3 realiza una descripción de los datos

1 Con información circa a 2018, incluye Argentina, Bolivia, Brasil, Chile, Colombia, Costa Rica, Ecuador, El Salvador, Guatemala, Honduras, México, Panamá, Paraguay, Perú, República Dominicana y Uruguay. [Acceso, marzo - 2020]. 
utilizados y presenta datos descriptivos de la evolución de la brecha de ingresos a lo largo de los 25 años analizados. La sección 4 describe las metodologías utilizadas para el cálculo de la brecha de ingresos laborales de género. La sección 5 presenta los resultados del estudio. La sección 6 presenta pruebas de consistencia. Finalmente, la sección 7 presenta las principales conclusiones del documento.

\section{REVISION DE LA LITERATURA}

Los avances de la región hacia la igualdad de género en los últimos años han sido propiciados por la mayor visibilidad de las mujeres en distintos ámbitos. Un punto de particular relevancia tienen que ver con la mayor participación de las mujeres en el mercado laboral a partir de $1980^{2}$ (Chioda, 2011). Sin embargo, esta participación no se da en igualdad de condiciones, las mujeres todavía tienen una mayor representación en puestos de trabajo mal remunerados, en trabajos informales y la brecha de ingresos laborales continúa siendo un tema relevante (Ñopo, 2012).

En lo que se refiere a la brecha de ingresos, la literatura ha tratado de distinguir la brecha de ingresos referente a las diferencias en características individuales y dotación de capital humano, de aquella no explicada y mayormente relacionada a prejuicios de género o discriminación (Atal et al., 2009).

Psacharopoulos y Tzannatos (1992) estudian la diferencia de ingresos laborales en 15 países de ALC a finales de los 80. Los autores encuentran evidencia de que las mujeres obtenían ingresos laborales por tan solo el $65 \%$ de lo que obtiene un hombre. Siendo que el porcentaje de la brecha de ingresos explicada por las diferencias en el nivel de educación y capital humano representaban solamente un tercio de la diferencia total, dejando una gran porción (alrededor del 43\%) de la diferencia sin explicación y posiblemente asociada a discriminación. Evidencia algo más reciente muestra que la brecha de ingresos se redujo desde 16\% hasta 9\% (Ñopo y Hoyos, 2010) entre 1992 y 2007. Sin embargo, la brecha no explicada representaba el 34\% en 1992 y el 30\%, en 2007. Es importante destacar que una parte significativa de la reducción de la brecha de ingresos en los últimos años se explica por el incremento de nivel educativo obtenido por las mujeres que ingresaron al mercado laboral (Chioda, 2011; Gasparini y Marchionni, 2015). Finalmente, la OIT (2019) en un análisis de 17 países y mediante la descomposición no paramétrica de Ñopo encuentran que la brecha no explicada de ingresos se redujo en el quinquenio 2012 y 2017 entre 2 y 3 puntos porcentuales (pp)

2 La evidencia sugiere que la inserción de la mujer al mercado laboral ha sido facilitada por el crecimiento económico de la región, el comercio, liberalización, urbanización, el incremento de nivel educacional obtenido y la reducción de la tasa de fertilidad. Gasparini y Marchionni (2015) indican que el incremento en la participación femenina en el mercado laboral de los países ALC también se puede explicar por el crecimiento económico de los 2000 y las políticas implementadas para incentivar y proteger el trabajo de las mujeres. 
y donde la brecha es más pronunciada entre los trabajadores de más bajos ingresos y donde las brechas son mayores para aquellos trabajadores autoempleados.

En el caso de Bolivia, existe alguna evidencia respecto de la evolución de la brecha de ingresos por género. Psacharopoulos y Tzannatos (1992), con datos de 1989, encontraron que las mujeres percibían el $62 \%$ del ingreso que percibe un hombre, donde solo el $15 \%$ de la brecha observada podía ser explicada por diferencias en la dotación en capital humano ${ }^{3}$. Rivero y Jiménez (1999) analizan las diferencias de ingreso en áreas urbanas de Bolivia entre 1981 y 1997, encuentran que la brecha de ingresos por género se redujo de $32 \%$ en 1981 a $11 \%$ en 1997. Asimismo, Contreras y Galván (2003) revisan la evolución de la discriminación contra mujeres e indígenas de Bolivia entre 1994 y 1999. En este estudio se confirma la existencia de discriminación de ingresos por género y etnicidad, siendo que el hecho de ser mujer e indígena incrementaba la brecha de ingresos entre 3,2 pp y 4 pp..$^{5}$ Nopo (2010), con datos de 2005 a nivel nacional, encuentra evidencia de que la brecha de ingresos no explicada podría llegar a ser tan alta como 17\%. Finalmente, OIT (2019) encuentra que la brecha no explicada de ingresos, siguiendo la descomposición de Ñopo, es de 30 pp para los trabajadores autoempleados y 8 pp para los trabajadores asalariados.

Sin embargo y a pesar de la evidencia recién expuesta, los estudios analizados utilizan modelos empíricos y grupos poblaciones de estudio diferentes, lo que imposibilita su comparación y por tanto el seguimiento de la evolución de la brecha de género. Siendo este hecho particularmente relevante para el diseño e implementación de políticas públicas que permitan acotar la brecha y permitir un acceso acorde a las necesidades de las mujeres al mercado laboral. El presente documento pretende contribuir a la literatura al proponer un análisis de la evolución de la brecha de género en Bolivia desde 1993 hasta 2018.

\section{DATOS Y ESTADISTICA DESCRIPTIVA}

\section{Datos}

Los datos utilizados en este documento provienen de las Encuestas de Hogares armonizadas por el Banco Interamericano de Desarrollo (BID) ${ }^{6}$. El presente documento utiliza 5 encuestas en el periodo de los 25 años analizados, según la disponibilidad de las mismas. Para esto, se utilizan datos de la Encuesta Integrada de Hogares 1993,

\footnotetext{
Información presentada en la tabla A-1 en el estudio de Psacharopoulos y Tzannatos (1992).

4 El estudio utiliza ecuaciones de Mincer corregidas para sesgo de selección y subsecuentemente una Descomposición de Fields.

5 Mediante su Descomposición de Fields.

6 Banco de datos de Encuestas de Hogares Armonizadas.
} 
posteriormente para 1999, 2006, 2013 y 2018 se utilizan las Encuestas de Hogares. Todas recolectadas por el Instituto Nacional de Estadística (INE).

Una característica importante para señalar tiene que ver con el hecho que las encuestas utilizadas en este documento presentan un diseño similar y el mismo nivel de representatividad. La única diferencia existente se da en la encuesta de 1993, que tiene representatividad solo a nivel de ciudades capitales. Para superar esta diferencia, el documento limita su ámbito de estudio al área urbana ${ }^{7}$, aunque se presentan resultados a nivel general en la sección 5. Un punto a destacar es el proceso de armonización de las encuestas de hogares que asegura la comparabilidad de las mismas en el transcurso de los años estudiados.

El presente análisis se restringe para personas entre 15 a 65 años y toma en cuenta únicamente información relacionada a la actividad económica primaria.

Como primer punto, se analiza la muestra de las cinco encuestas definidas para el presente estudio (Tabla 1), donde se observa que la encuesta de 1993 presenta 10.723 observaciones en el rango de edad a ser analizado. En 1999, la muestra se reduce

TABLA 1

NUMERO DE OBSERVACIONES POR ENCUESTA

\begin{tabular}{|l|c|c|c|c|c|c|c|c|c|c|c|}
\hline \multirow{2}{*}{} & \multicolumn{2}{|c|}{1993} & \multicolumn{2}{c|}{1999} & \multicolumn{2}{c|}{2006} & \multicolumn{2}{c|}{2013} & \multicolumn{2}{|c|}{2018} \\
\cline { 2 - 9 } & $\mathrm{N}$ & $\%$ & $\mathrm{~N}$ & $\%$ & $\mathrm{~N}$ & $\%$ & $\mathrm{~N}$ & $\%$ & $\mathrm{~N}$ & $\%$ \\
\hline Género \\
\hline Hombres & 5.487 & 46 & 2.038 & 47 & 3.197 & 47 & 8.309 & 47 & 8.908 & 47 \\
Mujeres & 6.402 & 54 & 2.294 & 53 & 3.630 & 53 & 9.269 & 53 & 9.853 & 53 \\
\hline Edad & $\begin{array}{l}\mid 7.476 \\
15-25\end{array}$ & 38 & 1.668 & 39 & 2.601 & 38 & 5.880 & 33 & 6096 & 32 \\
$26-35$ & 2.855 & 24 & 996 & 23 & 1.586 & 23 & 4.224 & 24 & 4458 & 24 \\
$36-45$ & 2.235 & 19 & 827 & 19 & 1.266 & 19 & 3.264 & 19 & 3721 & 20 \\
$46-55$ & 1.425 & 12 & 543 & 13 & 872 & 13 & 2.496 & 14 & 2552 & 14 \\
$56-65$ & 898 & 8 & 298 & 7 & 502 & 7 & 1.714 & 10 & 1934 & 10 \\
Total & 11.889 & 100 & 4.332 & 100 & 6.827 & 100 & 17.578 & 100 & 18.761 & 100 \\
\hline
\end{tabular}

Fuente: Elaboración propia a base de encuestas de hogares 1993, 1999, 2006, 2013 y 2018.

7 La Encuesta Integrada de Hogares 1993 únicamente consideraba ciudades capitales excluyendo a Cobija e incluyendo a la ciudad de El Alto, mientras que en 1999, 2006, 2013 y 2018 las encuestas tienen representación nacional. Considerando que para el presente documento restringimos el análisis a áreas urbanas, se sugiere precaución al comparar los resultados de 1993. 
a 4.444 observaciones, para 2006 se tienen 7.017 observaciones, en 2013 la cantidad de observaciones incrementó hasta 16.341 y finalmente en 2018 la encuesta recoge 19.210 observaciones en el rango etario definido. La reducción en el tamaño de muestra evidenciado entre 1993 y 1999 se explica por la decisión del INE de incluir al área rural e incluir secciones a la encuesta ${ }^{8}$. Desde 1999 hasta el 2018 el tamaño de muestra ha ido incrementándose gradualmente.

En promedio el 53\% de la muestra está conformado por mujeres y esta tendencia se mantiene para las cinco encuestas consideradas. La composición de edades es similar en todas las encuestas, aunque se observa un crecimiento en la participación de personas mayores de 55 años, coherente con el desarrollo demográfico del país.

\section{Estadística descriptiva}

A continuación se presenta información referente a la diferencia de ingresos por género en Bolivia. La Tabla 2 presenta la diferencia de ingresos entre hombres y mujeres a base del ingreso real por hora, diferenciando por algunas características demográficas. Para facilitar la comparación, los datos corresponden al ingreso por hora de las mujeres relativo al ingreso masculino (100\%) para cada categoría.

Se puede observar que, en relación con el ingreso de los hombres, en 1993, las mujeres ganaban 39\% menos. Esta diferencia se redujo hasta $10 \%$ en 2018, lo que nos presenta una primera evidencia de la reducción de la brecha de ingresos laborales en Bolivia.

Con relación a los rangos de edad, la menor diferencia entre hombres y mujeres se da en el rango de edad entre 26 y 35 años. Siendo esto cierto para todos los años analizados, aunque se puede evidenciar que la magnitud de la diferencia también se redujo sustancialmente. En 1993, una mujer entre 26 y 35 años obtenía solamente el $74 \%$ de lo que un hombre en ese rango etario, mientras que, en 2018, obtienen el $94 \%$ que el ingreso por hora de un hombre. Un hecho particularmente relevante a destacar es la reducción de las brechas de ingresos para todos los rangos etarios, incluso para aquellos entre 56 y 65 años, que es el rango de edad donde mayores diferencias de ingreso se observan.

Respecto del nivel educativo, se observa que las mujeres con educación terciaria pasaron de obtener el 60\% del ingreso por hora de un hombre en 1993 a recibir un ingreso similar (89\%) al de los hombres en 2018. Se evidencia también una reducción de la brecha para todos los niveles educativos, aunque aquellas mujeres que no tienen educación formal obtienen $17 \%$ menos que un hombre con la misma característica educativa.

8 La encuesta de 1999 fue ejecutada como parte del programa para el Mejoramiento de las Encuestas y la Medición de Condiciones de vida en América Latina y el Caribe (MECOVI). A partir de 1999 las encuestas de hogares en Bolivia tienen representación nacional. 
TABLA 2

INGRESO POR HORA DE LAS MUJERES RELATIVO AL INGRESO POR HORA DE LOS HOMBRES

\begin{tabular}{|c|c|c|c|c|c|}
\hline & $1993(\%)$ & $1999(\%)$ & $2006(\%)$ & $2013(\%)$ & $2018(\%)$ \\
\hline General & 61,4 & 82,8 & 63,0 & 81,8 & 89,6 \\
\hline \multicolumn{6}{|l|}{ Edad } \\
\hline $15-25$ & 60,0 & 69,6 & 48,6 & 81,9 & 84,3 \\
\hline $26-35$ & 74,4 & 84,2 & 73,2 & 79,9 & 94,1 \\
\hline $36-45$ & 61,3 & 90,7 & 64,0 & 82,1 & 87,8 \\
\hline $46-55$ & 57,6 & 75,7 & 62,2 & 85,6 & 95,5 \\
\hline $56-65$ & 39,5 & 87,5 & 69,8 & 75,2 & 78,9 \\
\hline \multicolumn{6}{|l|}{ Nivel de Educación } \\
\hline Ninguna & 62,7 & 78,2 & 70,0 & 67,2 & 82,7 \\
\hline Primaria & 54,8 & 67,1 & 76,0 & 76,7 & 86,5 \\
\hline Secundaria & 74,7 & 105,6 & 61,0 & 79,3 & 85,9 \\
\hline Terciaria & 60,4 & 79,7 & 63,5 & 90,2 & 88,9 \\
\hline \multicolumn{6}{|l|}{ Sector Económico } \\
\hline Minería e hidrocarburos & $\mathrm{n}, \mathrm{d}$, & 27,6 & $\mathrm{n}, \mathrm{d}$, & 82,6 & 77,6 \\
\hline Manufactura & 61,2 & 65,0 & 76,0 & 71,3 & 60,9 \\
\hline Construcción & $\mathrm{n}, \mathrm{d}$ & 47,4 & 37,7 & 85,0 & 83,5 \\
\hline Comercio & 57,1 & 83,4 & 61,7 & 74,8 & 78,2 \\
\hline Transporte y Almacenamiento & $\mathrm{n}, \mathrm{d}$, & $\mathrm{n}, \mathrm{d}$ & $\mathrm{n}, \mathrm{d}$ & 60,8 & 69,5 \\
\hline Servicios Financieros & 61,9 & 71,5 & 31,6 & 86,1 & 129,3 \\
\hline Servicios Sociales & 51,9 & 75,3 & 64,3 & 82,7 & 92,2 \\
\hline \multicolumn{6}{|l|}{ Ocupación } \\
\hline Profesionales, Técnicos y Directivos & 74,2 & 94,1 & 57,5 & 94,5 & 92,7 \\
\hline Administrativos & 88,5 & 88,7 & 87,1 & 95,6 & 110,0 \\
\hline Comerciantes y Vendedores & 48,0 & 86,5 & 61,6 & 59,4 & 77,0 \\
\hline Servicios & 52,8 & 103,4 & 90,6 & 101,0 & 99,8 \\
\hline Trabajadores No Agropecuarios & 59,2 & 49,9 & 72,5 & 60,6 & 68,7 \\
\hline Fuerzas Armadas y Policía & n.d. & n.d. & 54,9 & $\mathrm{n}, \mathrm{d}$ & 69,3 \\
\hline \multicolumn{6}{|l|}{ Formalidad } \\
\hline Informal & n.d. & 75,4 & 60,0 & 74,4 & 84,1 \\
\hline Formal & n.d. & 112,4 & 83,4 & 98,9 & 103,6 \\
\hline
\end{tabular}

Fuente: Elaboración propia a base de encuestas de hogares de 1993, 1999, 2006, 2013 y 2018.

Nota: Servicios Sociales incluye las actividades de electricidad, agua y gas.

n.d. No Disponible. Cuando los datos disponibles no son suficientes para calcular el porcentaje.

Por sector económico, en 2018, los sectores de servicios financieros, construcción y servicios son los sectores donde los ingresos entre hombres y mujeres son iguales, mientras que, en el sector de comercio y manufactura, todavía persisten diferencias de más de 20 pp. Nuevamente, es importante destacar la evolución en la brecha de ingresos, particularmente en los sectores de servicios financieros y sociales a lo largo de los 25 años analizados. Por ocupación, los directivos, profesionales, técnicos y 
servicios administrativos son las ocupaciones que presentan mayores niveles de equidad de ingresos. En 1993 una mujer directiva, profesional o técnico ganaba el $74 \%$ de lo que ganaba un hombre, mientras que en 2018 obtenía un ingreso similar por hora trabajada $(93 \%)$.

Finalmente, y con relación a la formalidad ${ }^{9}$ de los empleos a los que acceden los trabajadores en Bolivia, las mujeres que se desempeñan en trabajos formales obtienen un ingreso igual o superior al de los hombres. La penalidad de ingresos para las mujeres en el sector informal pasó de aproximadamente el 25\% en 1999 hasta $16 \%$ en 2018.

\section{ESTRATEGIA EMPIRICA}

\section{Descomposición Blinder y Oaxaca}

A continuación se cuantificará y analizará la evolución de la brecha de género en Bolivia en los últimos 25 años. Para hacer esto, se analizará la porción de la brecha de ingresos laborales que puede explicarse por diferencias en el capital humano (llámese educación, experiencia laboral, etc.) y aquella parte que no puede ser explicada y estaría asociada a discriminación de género ${ }^{10}$.

La literatura usualmente ha afrontado esta pregunta utilizando la descomposición de Blinder-Oaxaca. El método Blinder-Oaxaca utiliza ecuaciones de salario del tipo Mincer para dividir la diferencia de ingresos laborales en dos grupos, uno que se puede “explicar" por diferencias grupales y características productivas como la educación o la experiencia laboral y un segundo componente residual, no explicado, generalmente asociado a discriminación (Jann, 2008).

La racionalidad formal de la metodología es la que sigue: dados dos grupos, hombres $(\mathrm{H})$ y mujeres $(\mathrm{M})$; un resultado de interés que es nuestra variable Y (en este caso el logaritmo del ingreso por hora); y un grupo de variables explicativas $\mathrm{X}$ (educación, experiencia, etc.). La pregunta de interés a responder es ¿Cuánto de la diferencia media del ingreso laboral se debe a diferencias grupales en los predictores X?:

9 En el presente documento se considera formal aquel trabajador que tiene acceso a los beneficios de seguridad de largo plazo (pensiones).

10 Varios modelos económicos han tratado formalmente la oferta y demanda de los factores que pueden explicar la brecha de ingresos en las minorías. Becker (1957) por ejemplo propone una teoría conocida como Taste for Discrimination donde la discriminación se debe a un prejuicio personal o un "gusto" personal en contra de asociarse con un grupo en particular. Consecuentemente, los empleadores actúan como si hubiera un costo no económico por emplear a un grupo en particular. Otra explicación común es la discriminación estadística que describe a la discriminación como el acto de agentes racionales de utilizar características grupales agregadas para evaluar características individuales, esto implica que agentes de distintos grupos pueden ser tratados de forma diferenciada. Por ejemplo, si las empresas creen que las mujeres en edad fértil tienen una probabilidad mayor de pausar sus carreras por tener hijos, pagarían menores sueldos a las mujeres en este rango de edad para mitigar la probabilidad de perder a una trabajadora (Hoyos et al., 2010). 


$$
E G a p=E\left(Y_{H}\right)-E\left(Y_{M}\right)
$$

Donde $E\left(Y_{g}\right)$ denota la esperanza de la variable de interés. Utilizando una ecuación tipo Mincer para explicar el ingreso, podemos desagregar el mismo tal que $Y_{g}=\alpha_{g}+\sum_{i=1}^{k} X_{i k} \beta_{g i k}+\epsilon_{g i}$, donde $g$ describe si la ecuación se realiza a hombres (H) o mujeres (M). La expresión puede ser reemplazada en la ecuación [1], tal que:

$$
\begin{gathered}
\text { EGap }=E\left(\alpha_{H}+\sum_{i=1}^{k} X_{i k} \beta_{H i k}+\epsilon_{H i}\right)-E\left(\alpha_{M}+\sum_{i=1}^{k} X_{i k} \beta_{M i k}+\epsilon_{M i}\right) \\
\text { EGap }=\hat{\alpha}_{H}+\sum_{i=1}^{k} \bar{X}_{i k} \hat{\beta}_{H i k}-\hat{\alpha}_{M}-\sum_{i=1}^{k} \overline{X_{i k}} \hat{\beta}_{M i k}
\end{gathered}
$$

Reordenando algunas variables podemos identificar la contribución de las diferencias grupales en los predictores al resultado final.

$$
E G a p=\left(\hat{\alpha}_{H}-\hat{\alpha}_{M}\right)+\sum_{i=1}^{k} \bar{X}_{i k}\left(\hat{\beta}_{H i k}-\hat{\beta}_{M i k}\right)+\sum_{i=1}^{k}\left(\bar{X}_{H i k}-\bar{X}_{M i k}\right) \hat{\beta}_{H i k}
$$

Donde el último componente de la ecuación [4] corresponde a la brecha de ingresos explicada por las diferencias observables, mientras que los dos primeros componentes corresponden a las diferencias no observables.

La descomposición de Blinder-Oaxaca es el método mayormente utilizado para el cálculo de brechas de ingreso, sin embargo, presenta algunas limitaciones que son relevantes de mencionar. Primero, la relación entre características e ingresos no necesariamente podría ser lineal como asume el modelo Blinder-Oaxaca. Segundo, el modelo es únicamente informativo acerca de la descomposición de la brecha de ingresos. Tercero, el método no logra restringir su comparación a individuos comparables, debido a las diferencias en el soporte de la distribución empírica de las características de los individuos (Ñopo, 2012).

\section{Descomposición de Ñopo}

Como respuesta a las limitaciones, Ñopo (2008) desarrolló un método de descomposición no paramétrico. El método responde a la misma pregunta contrafactual, considerando las diferencias a lo largo de la distribución de ingresos y no solo en el promedio, como lo hace Blinder-Oaxaca.

En este sentido, la metodología de Ñopo restringe la comparación de diferencias únicamente entre hombres y mujeres con características comparables. Por lo que crea una muestra sintética de individuos al emparejar hombres y mujeres con características observables idénticas y así no necesita asumir ninguna forma funcional entre la 
relación entre características e ingresos. El emparejamiento se realiza por medio de características discretas, de este modo, el emparejamiento no necesita el uso de Propensity Score u otra noción de distancia entre las características de hombres y mujeres (Ñopo 2008).

Este procedimiento crea tres juegos de individuos: i) aquellas mujeres y hombres que están emparejados (soporte común); ii) aquellas mujeres con características observables para las que no existen hombres comparables (conocido según la metodología como Maid Effect) y, iii) aquellos hombres para los que no existen mujeres comparables (conocido como CEO Effect). Por construcción, aquellos hombres y mujeres en el soporte común no presentan características observables diferentes y en consecuencia las diferencias en ingresos, si es que existieran, no se podrían explicar por estas características.

En este sentido, el procedimiento descompone la brecha de ingreso en cuatro elementos:

$$
\delta=\delta_{X}+\delta_{H}+\delta_{M}+\delta_{0}
$$

El primero explica la diferencia de ingreso en relación con las características observables $\left(\delta_{X}\right)$, el segundo y tercer elementos explican la diferencia de aquellos individuos fuera del soporte común $\left(\delta_{H}\right.$ y $\left.\delta_{M}\right)$ y el último elemento que puede ser interpretado como discriminación $\left(\delta_{0}\right)$. Un punto importante a notar es el hecho de que el componente no explicado de Ñopo es comparable al componente no explicado en el modelo Blinder-Oaxaca, solo que restringido al soporte común dentro de la distribución.

A pesar de que la metodología de descomposición de Ñopo tiene atributos importantes que corrigen limitaciones específicas del modelo Blinder-Oaxaca, también presentan sus propias limitaciones que se deben mencionar. Primero, debido a que el emparejamiento se construye con variables discretas, la probabilidad de encontrar una persona con las mismas características y dotaciones para cada hombre o mujer disminuye a medida que se incrementa el número de variables incluidas (Enamorado et al., 2009), lo que reduce el soporte común si el análisis se realiza con una cantidad importante de características observables. Este fenómeno se conoce en la literatura como la Maldición de Dimensión. En general, el tamaño del soporte común estará restringido en función al número de variables a incluirse en el análisis.

Un punto que atañe a ambas metodologías, es el hecho que tanto la descomposición de Ñopo como la de Blinder-Oaxaca solamente consideran características observables, omitiendo aquellas características no observables como son el esfuerzo, interés por participar en el mercado laboral o responsabilidades del hogar, lo que posiblemente genere estimaciones sesgadas por omisión de variables. El resultado de nuevas investigaciones referentes a la participación de las mujeres en el mercado laboral encuentran evidencia de que las preferencias y actitudes entre hombres y mujeres hacia el trabajo no son necesariamente idénticas (Chioda, 2011). 
El presente documento empleará ambas metodologías en el análisis de la evolución de la brecha de ingresos entre hombres y mujeres en Bolivia. Utilizar ambas metodologías permite aprovechar las ventajas metodológicas de cada una para tratar de entender la evolución y sus posibles explicaciones. Asimismo, utilizar ambas metodologías permite crear un marco metodológico comparable con los estudios existentes y con futuras actualizaciones, independientemente de la metodología que se utilice.

\section{RESULTADOS}

Considerando que el presente documento utiliza ambos métodos de evaluación, se utilizarán las mismas variables explicativas tanto para para la descomposición Blinder-Oaxaca como para la descomposición de Nopo. Se consideran las siguientes variables: años de educación, experiencia laboral estimada ${ }^{11}$; experiencia laboral al cuadrado; una variable dummy para cada grupo educacional (existen cuatro grupos: sin educación o inferior a primaria, primaria completa, secundaria completa y terciaria, el grupo sin educación es el grupo base); una variable dummy para cada grupo de edad (15-25, 26-35, 36-45 y de 56-65, el grupo entre 45 y 56 es el grupo base); una variable dummy que toma el valor de 1 si la persona está casada; una variable dummy por cada sector económico; una dummy que toma el valor de 1 si trabaja formalmente; y una dummy que toma el valor de 1 ante la presencia de menores a 6 años en el hogar. Por comparabilidad con la descomposición de Ñopo, no se incluye el grupo ocupacional en este primer análisis, aunque sí más adelante.

La Tabla 3 presenta los resultados del cálculo de la brecha de ingreso por hora utilizando el modelo Blinder-Oaxaca. Los resultados muestran que la diferencia en el ingreso por hora por género en 1993 era 32\% del ingreso laboral promedio de los hombres, de esta diferencia, la parte explicada representa tan solo el $11 \%$ mientras que la parte sin explicación representa el $20 \%{ }^{12}$. Donde la porción de la brecha explicada corresponde al mayor nivel educativo y de experiencia laboral de los hombres. En 1999, la diferencia de ingresos fue de aproximadamente $21 \%$, donde la parte no explicada de la brecha era de $15 \%$. En 2006, la brecha de ingresos reduce hasta $17 \%$, del que $12 \%$ no era explicada por características observables. En 2013, la diferencia continuó su tendencia decreciente hasta $11 \%$, siendo $8 \%$ que corresponde al componente no explicado. Finalmente, para el 2018, la brecha representaba solamente el $7 \%$ de los ingresos, de estos, $6 \%$ corresponde al componente no explicado.

11 Calculado como Experiencia=edad-años de educación-6.

12 La brecha de género está calculada como Diferencia/Estimación Hombre. La brecha con explicación se calcula como "Explicada"/Estimación Hombre. La brecha no explicada se calcula como "No Explicada"/ Estimación Hombre. 


\section{TABLA 3}

DESCOMPOSICION BLINDER-OAXACA

\begin{tabular}{|c|c|c|c|c|c|}
\hline & \multicolumn{5}{|c|}{ Logaritmo del ingreso por hora } \\
\hline & 1993 & 1999 & 2006 & 2013 & 2018 \\
\hline \multicolumn{6}{|l|}{ Diferencial } \\
\hline Estimación Hombre & $\begin{array}{c}1,696^{* * * *} \\
(116,29)\end{array}$ & $\begin{array}{c}1,973^{* * *} \\
(76,61)\end{array}$ & $\begin{array}{l}1,921^{* * * *} \\
(98,33)\end{array}$ & $\begin{array}{l}2,200^{* * * *} \\
(214,02)\end{array}$ & $\begin{array}{l}2,258^{* * * *} \\
(268,25)\end{array}$ \\
\hline Estimación Mujer & $\begin{array}{c}1,158 * * * \\
(59,30)\end{array}$ & $\begin{array}{c}1,560 * * * \\
(42,10)\end{array}$ & $\begin{array}{c}1,594 * * * \\
(60,87)\end{array}$ & $\begin{array}{l}1,964 * * * \\
(140,61)\end{array}$ & $\begin{array}{c}2,095 \text { **** } \\
(166,14)\end{array}$ \\
\hline Diferencia & $\begin{array}{c}0,538 * * * \\
(22,08) \\
\end{array}$ & $\begin{array}{c}0,413 * * * \\
(9,15) \\
\end{array}$ & $\begin{array}{c}0,327 * * * \\
(10,02) \\
\end{array}$ & $\begin{array}{c}0,235^{* * * *} \\
(13,58) \\
\end{array}$ & $\begin{array}{c}0,163 * * * \\
(10,72) \\
\end{array}$ \\
\hline \multicolumn{6}{|l|}{ Descomposición } \\
\hline Explicada & $\begin{array}{c}0,191 * * * \\
(10,04)\end{array}$ & $\begin{array}{c}0,120 * * * \\
(3,69)\end{array}$ & $\begin{array}{c}0,0903 * * * \\
(4,05)\end{array}$ & $\begin{array}{c}0,0662 * * * \\
(5,91)\end{array}$ & $\begin{array}{c}0,0291 * * \\
(2,83)\end{array}$ \\
\hline No Explicada & $\begin{array}{c}0,347 * * * \\
(14,63)\end{array}$ & $\begin{array}{c}0,293 * * * * \\
(6,57)\end{array}$ & $\begin{array}{c}0,237 * * * \\
(7,43)\end{array}$ & $\begin{array}{c}0,169^{* * * *} \\
(9,83)\end{array}$ & $\begin{array}{c}0,133^{* * * *} \\
(8,51)\end{array}$ \\
\hline \multicolumn{6}{|c|}{ Descomposición (como porcentaje del ingreso laboral por hora para los hombres) } \\
\hline Total & $32 \%$ & $21 \%$ & $17 \%$ & $11 \%$ & $7 \%$ \\
\hline Explicada & $11 \%$ & $6 \%$ & $5 \%$ & $3 \%$ & $1 \%$ \\
\hline No Explicada & $20 \%$ & $15 \%$ & $12 \%$ & $8 \%$ & $6 \%$ \\
\hline Observaciones & 6.313 & 2.330 & 3.807 & 10.418 & 10.603 \\
\hline
\end{tabular}

Estadístico t en paréntesis

$* p<0.05,{ }^{* *} p<0.01,{ }^{* * *} p<0.001$

Fuente: Elaboración propia a base de los datos de las encuestas de hogares 1993, 1999, 2006, 2013 y 2018.

Es interesante destacar la disminución de la brecha de ingresos por género en los últimos 25 años, pasando de 32\% en 1993 a 7\% en el 2018. Destaca la reducción del porcentaje explicado de la brecha que pasó de $11 \%$ a $1 \%$, aunque también la reducción del componente no explicado, que se redujo de $20 \%$ a $6 \%$.

Para analizar las causas de la reducción de la brecha explicada, la Tabla 4 presenta una desagregación de los principales componentes que explican las diferencias entre hombres y mujeres. Para esto se agregan las variables relacionadas a características educativas, aquellas relacionadas a la experiencia, aquellas relacionadas a características personales y familiares, el sector de actividad, la región y la formalidad. Destaca la reducción de la porción explicada de la brecha asociada a diferencias educacionales, las que pasaron de ser las más relevantes a no ser significativas. Este se debe a un mayor acceso de mujeres con mayores niveles de educación al mercado laboral, como se puede evidenciar en la Tabla A1. Asimismo, se observa que las características familiares dejan de ser relevantes para explicar la diferencia de ingresos, lo que hace referencia al impacto de los cambios culturales y de roles en el país, ser una mujer 
TABLA 4

COMPONENTES DE LA DIFERENCIA EXPLICADA - BLINDER-OAXACA

\begin{tabular}{|l|c|c|c|c|c|}
\hline & \multicolumn{5}{|c|}{ Logaritmo del ingreso por hora } \\
\cline { 2 - 6 } & 1993 & 1999 & 2006 & 2013 & 2018 \\
\hline Diferencia Explicada & $0,191^{* * *}$ & $0,120^{* * *}$ & $0,090^{* * *}$ & $0,066^{* * *}$ & $0,029^{* *}$ \\
Educación & $0,113^{* * *}$ & $0,091^{* * *}$ & $0,041^{* *}$ & $0,016^{* *}$ & $-0,005$ \\
Experiencia & $-0,007$ & $-0,020$ & $-0,013$ & $-0,004$ & 0,001 \\
Características & $0,021^{* * *}$ & 0,009 & $0,017^{* *}$ & $0,013^{* * *}$ & 0,002 \\
personales y familiares & $0,055^{* * *}$ & 0,034 & $0,028^{* *}$ & $0,036^{* * *}$ & $0,026^{* * *}$ \\
Sector de Actividad & 0,009 & 0,000 & 0,002 & $-0,002$ & 0,000 \\
Región & n.d. & 0,007 & $0,015^{* *}$ & $0,007^{* *}$ & 0,004 \\
Sector Formal & \multicolumn{7}{|c}{} & & \\
\hline
\end{tabular}

$* p<0.05,{ }^{* *} p<0.01,{ }^{* * *} p<0.001$

Fuente: Elaboración propia a base de los datos de las encuestas de hogares 1993, 1999, 2006, 2013 y 2018.

casada deja de ser relevante para explicar las diferencias de ingreso. De la misma forma, es importante destacar que el sector de actividad donde se desempeñan las mujeres sigue siendo relevante para explicar la diferencia de ingresos y que nos da luces acerca de segregación sectorial. En Anexos se presenta el mismo análisis incluyendo la variable de ocupación (Tabla A2.), donde también se evidencia un menor acceso de mujeres a las ocupaciones con mayores ingresos. Estas afirmaciones se pueden contrastar en la sección en el Anexo 2, que presenta una metodología para la descomposición de ingresos a base de las variables explicativas utilizadas conocida como Descomposición de Fields.

La Tabla 5 presenta los resultados de la descomposición Ñopo. Si bien se utiliza el mismo set de variables explicativas, aunque considerando que la metodología de Ñopo emplea datos discretos para el emparejamiento, se incluyen variables de grupo de niveles de educación en lugar de la variable años de educación. Considerando la Maldición de Dimensión, porque la inclusión de variables explicativas tiene un impacto negativo en el soporte común, el resultado no incluye la variable de ocupación laboral, como en el caso de la Descomposición de Blinder-Oaxaca recién reportado. En la sección 5 se presentará los resultados incluyendo esta variable.

La primera columna de la Tabla 5 muestra que la brecha de ingreso por género en 1993 era de $43 \%$ del ingreso promedio de los hombres, siendo la parte no explicada de $31 \%$. El componente explicado es $8 \%$. El CEO Effect (hombres para los cuales el procedimiento no halló mujeres con las mismas características) es positivo en la brecha de género y es igual a 5\%. Asimismo, el Maid Effect (mujeres para las que no hay hombres con las mismas características) es negativo (-0,4\%). Es interesante notar que el porcentaje de hombres y mujeres en el soporte común es de 55\% y $72 \%$, respectivamente, de acuerdo con lo que se esperaba debido a la metodología de emparejamiento. 


\section{TABLA 5}

DESCOMPOSICION ÑOPO

\begin{tabular}{|l|c|c|c|c|c|}
\hline & $1993(\%)$ & $1999(\%)$ & $2006(\%)$ & $2013(\%)$ & $2018(\%)$ \\
\hline$\delta($ Total $)$ & 43 & 28 & 23 & 13 & 7 \\
$\delta_{0} \delta_{0}$ (No explicada) & 31 & 21 & 16 & 11 & 5 \\
$\delta_{\mathrm{M}} \delta_{\mathrm{M}}$ (Maid Effect) & -0 & -4 & -1 & -2 & -2 \\
$\delta_{\mathrm{F}} \delta_{\mathrm{F}}($ CEO Effect) & 5 & 4 & 2 & 2 & 1 \\
$\delta_{\mathrm{X}} \delta_{\mathrm{X}}$ (Explicado) & 8 & 8 & 6 & 2 & 2 \\
\hline$\%$ Hombres & 55 & 36 & 41 & 57 & 53 \\
$\%$ Mujeres & 72 & 53 & 58 & 76 & 78 \\
\hline Error Estándar & 2 & 4 & 3 & 1 & 1 \\
\hline Error Estándar $\delta_{0}$ & \multicolumn{5}{|l}{}
\end{tabular}

Fuente: Elaboración propia a base de los datos de las encuestas de hogares 1993, 1999, 2006, 2013 y 2018.

Siguiendo el análisis, en 1999 la brecha se redujo a $28 \%$ con un componente no explicado de $21 \%$ y un componente explicado igual a $8 \%$. El CEO Effect fue de $4 \%$ y el Maid Effect negativo e igual a $-4 \%$. El 36\% de los hombres y 53\% de las mujeres se encontraban en el soporte común.

Para el 2006 la brecha tuvo una nueva reducción a 23\% con un componente no explicado de $16 \%{ }^{13}$, un CEO Effect de $2 \%$ y un Maid Effect igual a $-1 \%$ En 2013, la brecha de ingresos llegó a ser $13 \%$ del ingreso promedio, compuesto básicamente por el componente no explicado o de discriminación $11 \%$.

Finalmente, para el 2018, la brecha de ingresos se redujo a la mitad y llego a 7\% del ingreso promedio. La parte no explicada de la brecha es de 5\%. En este caso, el 53\% de los hombres así como 78\% de las mujeres se encontraban en el soporte común.

Dos resultados destacan en particular del presente análisis. Primero, la descomposición Ñopo muestra un proceso de cierre de brecha en el periodo de 43\% a 7\%. Segundo, la reducción en la brecha explicada por diferencias observables es consistente con los datos del análisis de Blinder-Oaxaca (BO), reduciéndose de $8 \%$ a 2\%. También es importante destacar la reducción del CEO Effect, la parte de la diferencia explicada por hombres con características no replicables para las mujeres, componente que tradicionalmente se asocia a la presencia de hombres en altos cargos jerárquicos, se redujo, lo que podría interpretarse como una señal de un mejor acceso de mujeres a puestos gerenciales. Sin embargo, datos del Instituto Nacional de Estadística, en su reporte de remuneraciones del sector privado, muestran una menor participación de mujeres en puestos gerenciales, lo que coincide con datos provenientes de la Encuesta

13 Este porcentaje es similar al 17\% que encuentra Ñopo (2010) para el año 2005. 
de Demanda Laboral en Bolivia ${ }^{14}$, donde también se evidencia que las empresas siguen prefiriendo mayoritariamente a hombres para puestos gerenciales.

El Gráfico 1 presenta el resumen del total de la brecha por género encontrada bajo el cálculo de ambas metodologías para los 25 años analizados, el resumen incluye tanto el componente explicado y el componente no explicado. Destaca la convergencia de la brecha con ambas metodologías.

\section{GRAFICO 1}

\section{BRECHA DE INGRESO TOTAL ESTIMADA MEDIANTE LA METODOLOGIA DE BLINDER-OAXACA Y ÑOPO}

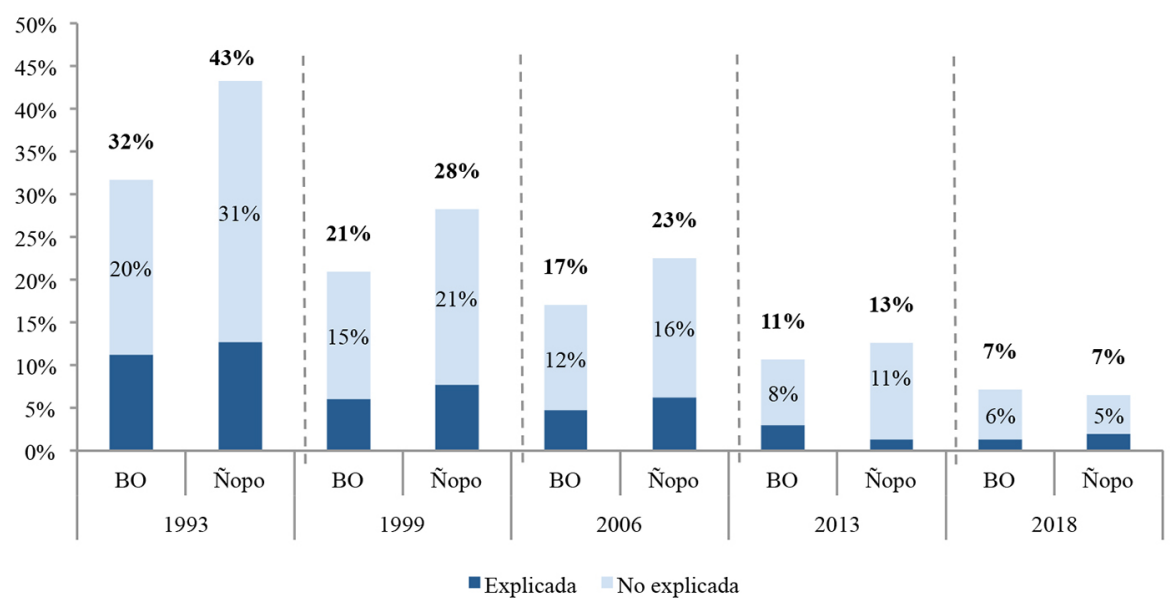

Fuente: Elaboración propia utilizando datos de encuestas de hogares de 1993, 1999, 2006, 2013 y 2018. Las barras presentan el componente explicado y no explicado de la brecha de ingresos por género. Para la metodología de Ñopo, el dato del componente explicado se calcula como la sumatoria del componente explicado, el CEO Effect y el Maid Effect.

A continuación se presenta la evolución de la brecha de ingresos no explicada en el periodo analizado empleando ambos procedimientos. El Gráfico 2 presenta la brecha no explicada incluyendo variaciones 2 desviaciones estándar arriba y abajo del estimador. Los resultados nos muestran una evolución robusta de la brecha no explicada con ambas metodologías, donde destaca que las diferencias entre ambos estimadores no son estadísticamente diferentes, con excepción del año 1993.

14 Encuesta recolectada por el BID en Bolivia entre 2015 y 2016. Urquidi et al. (2018) "Encuesta Mercado Laboral: Oferta 2016, Demanda 2015-2016". 


\section{GRAFICO 2}

\section{BRECHA DE INGRESO NO EXPLICADA COMPARATIVA}

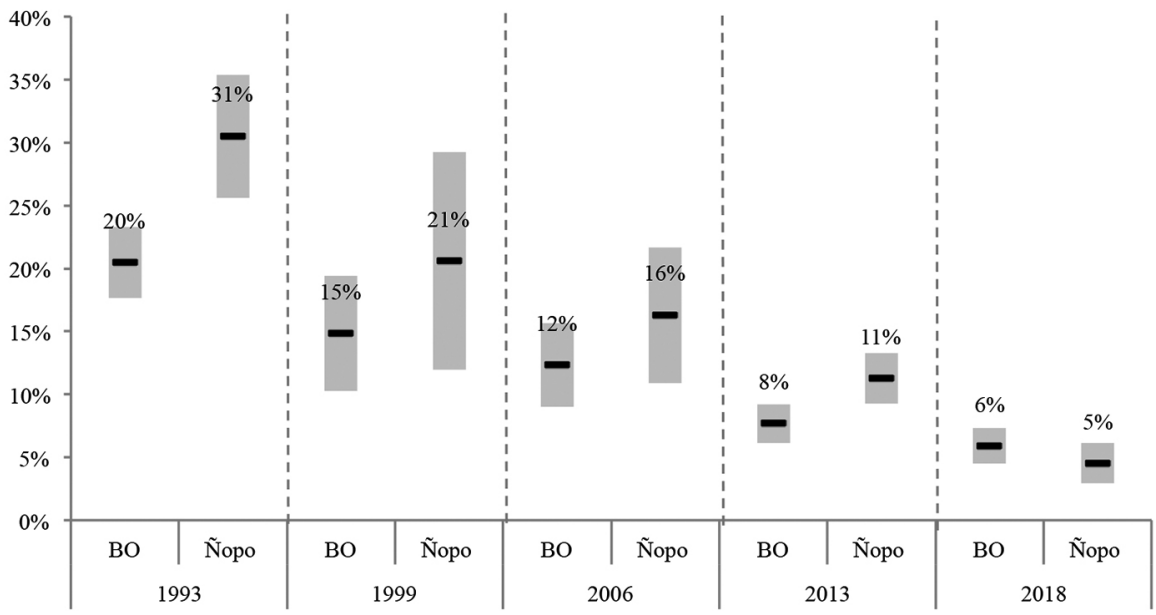

Fuente: Elaboración propia utilizando datos de encuestas de hogares de 1993, 1999, 2006, 2013 y 2018. Las barras presentan el componente no explicado al $95 \%$ de nivel de confianza.

La descomposición de Ñopo permite explorar de forma desagregada la brecha de ingresos. El Gráfico 3 presenta la diferencia en la brecha de género por nivel de educación. Se observa que la brecha no explicada de ingresos se reduce a mayor nivel de educación: mientras que la brecha de ingresos era de $47 \%$ y $54 \%$ para aquellas mujeres sin educación y con educación primaria en 1993, la brecha alcanzaba a $23 \%$ y $12 \%$ si se compara aquellas mujeres con estudios secundarios y terciarios, respectivamente. En 2018, la brecha no explicada para las mujeres sin educación era de $5 \%$, para las mujeres con educación primaria y secundaria $7 \%$ y $6 \%$, respectivamente, y para aquellas mujeres con educación terciaria, la brecha de ingresos era de apenas 2\%. Vale la pena destacar que en 1993 la brecha no explicada se reducía drásticamente al nivel de educación secundaria, mientras que en 2018, la brecha cae para aquellas mujeres con estudios terciarios. Este fenómeno se puede explicar por el incremento del nivel educativo de la fuerza laboral.

Respecto de la evolución del componente no explicado de la brecha de ingresos laborales por grupo etario, se puede evidenciar una mayor discriminación para aquellas trabajadoras en las colas de la distribución de edad, los jóvenes entre 15 y 25 años y las personas más cercanas a la edad de jubilación (56 a 65 años) presentan mayores diferencias no explicadas en los ingresos (Gráfico 4). 


\section{GRAFICO 3}

\section{BRECHA DE INGRESOS NO EXPLICADA POR NIVEL DE EDUCACION MEDIANTE LA DESCOMPOSICION DE ÑOPO}

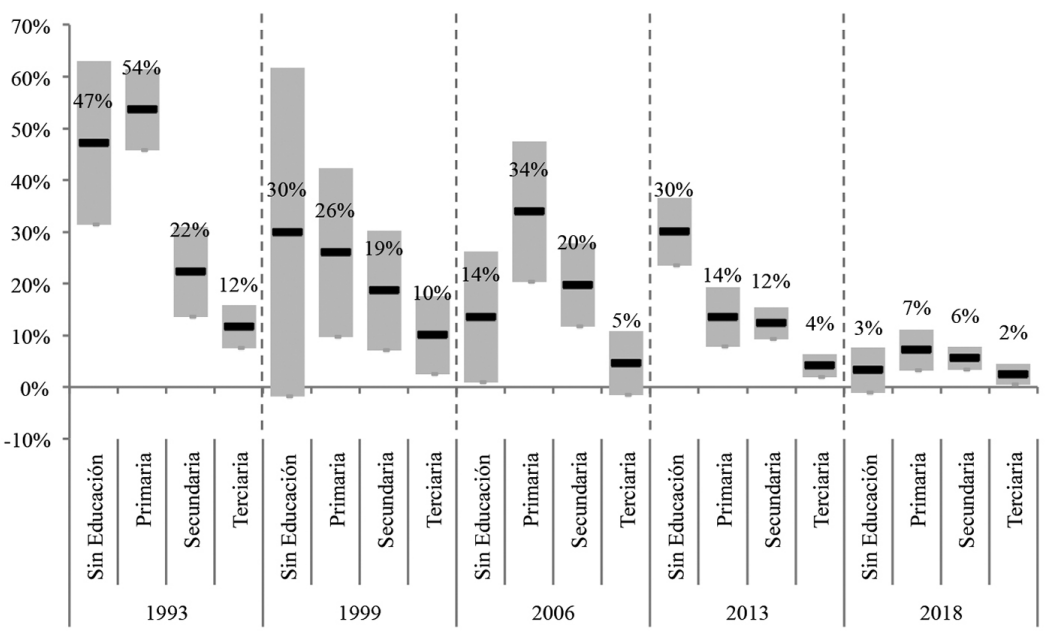

Fuente: Elaboración propia utilizando datos de encuestas de hogares de 1993, 1999, 2006, 2013 y 2018.

Las barras presentan el componente no explicado al $95 \%$ de nivel de confianza.

\section{GRAFICO 4}

\section{BRECHA DE INGRESOS NO EXPLICADA POR EDAD MEDIANTE LA DESCOMPOSICION DE ÑOPO}

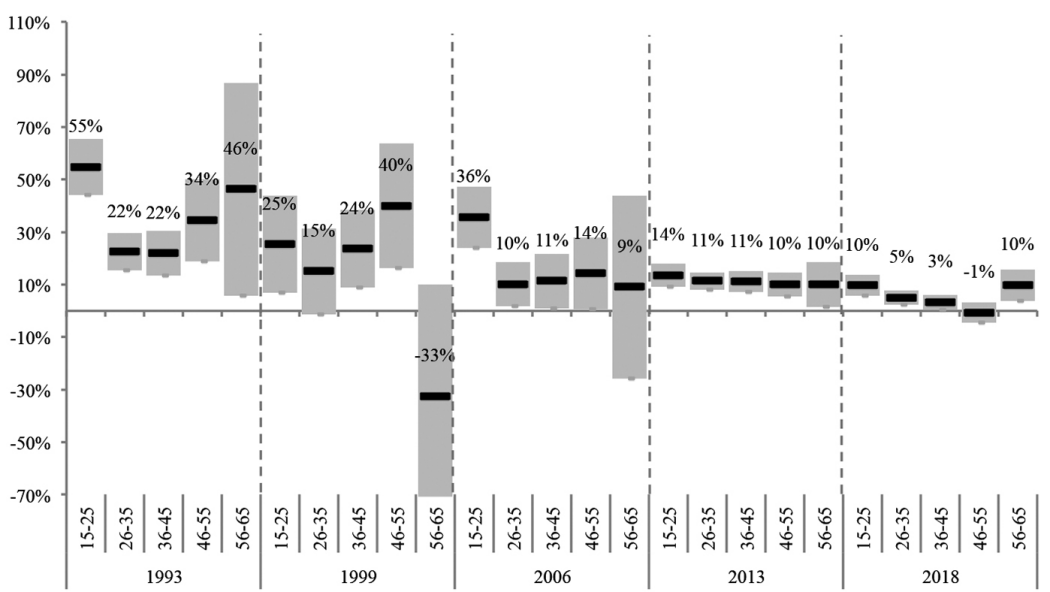

Fuente: Elaboración propia utilizando datos de encuestas de hogares de 1993, 1999, 2006, 2013 y 2018. Las barras presentan el componente no explicado al $95 \%$ de nivel de confianza. 
Desagregando el impacto de la brecha no explicada por formalidad, se observa que la brecha no explicada es mucho mayor en el sector informal. El análisis al respecto no incluye información para 1993, debido a que la encuesta no disponía información que permita calcular de forma homogénea la definición de informalidad (acceso a seguridad social de largo plazo) como en las otras encuestas. La brecha no explicada se redujo desde 7\% en el sector formal en 1999 hasta $0 \%$ en 2018. Para el sector informal la brecha no explicada era tan alta como $27 \%$ en 1999 , se redujo hasta $22 \%$ en 2006, pasando a 18\% en 2013 y finalmente a 7\% en 2018 (Gráfico 6, panel derecho). Es importante destacar que la brecha total de ingresos es negativa en el sector formal (Gráfico 5, panel izquierdo), debido a la presencia de mujeres con mayor nivel educacional en el sector formal y consecuentemente con mejores trabajos.

\section{GRAFICO 5}

\section{BRECHA DE INGRESOS POR FORMALIDAD MEDIANTE LA DESCOMPOSICION NOOPO BRECHA TOTAL (IZQ.), BRECHA NO EXPLICADA (DER.)}
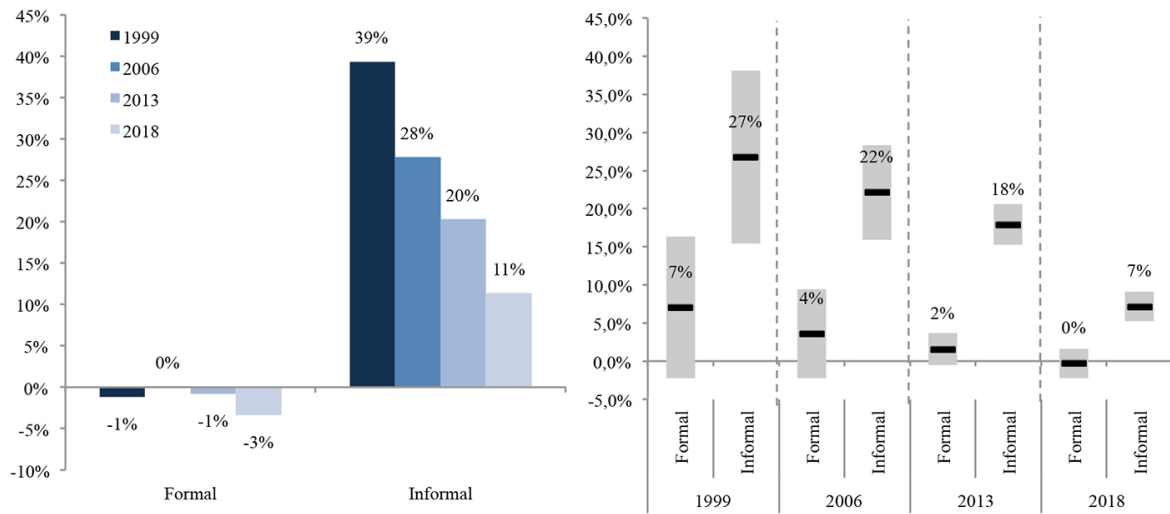

Fuente: Elaboración propia utilizando datos de encuestas de hogares de 1993, 1999, 2006, 2013 y 2018. Las barras presentan el componente no explicado al $95 \%$ de nivel de confianza.

Respecto de la brecha de ingresos en el sector informal, es importante recordar el alto nivel de informalidad en Bolivia que alcanza a $80 \%$ de la población ocupada, siendo $73 \%$ en el área urbana. Considerando esto, se puede desagregar el análisis de informalidad en dos grupos. El primero corresponde a los asalariados informales, donde la brecha de ingresos no explicada se redujo desde $23 \%$ en 1999 hasta $4 \%$ en 2018 , la brecha podría estar explicada por un menor control gubernamental por las características de este tipo de trabajo, que ya de por sí no tiene acceso a seguridad social (Gráfico A1). El segundo grupo corresponde a los informales por cuenta propia, que son el grupo (de estos analizados) con mayores niveles de brecha no explicada, 
el que pasó de 29\% en 1999 a 9\% en 2018, las explicaciones de estas diferencias se pueden entender por una sobrerrepresentación de las mujeres en las ocupaciones que generan menores ingresos (Tabla A3).

\section{PRUEBA DE ROBUSTEZ}

Para evaluar la validez de los resultados se analiza el efecto respecto de las estimaciones incluyendo al área rural y la variable omitida de ocupación, en el caso de la Descomposición Ñopo.

El Gráfico 6 presenta los resultados de la descomposición de ingresos no explicada mediante la metodología de Blinder-Oaxaca después de incluir el área rural. Se puede observar que la inclusión del área rural tiene un impacto significativo en la brecha estimada. Como se esperaba, la inclusión del área rural incrementa la brecha no explicada en 1999 de $15 \%$ a $24 \%$, de $12 \%$ a $16 \%$ en el 2006, de $8 \%$ a $10 \%$ el 2013 y de $6 \%$ a $8 \%$ en $2018{ }^{15}$. La brecha de ingresos es mayor en las áreas rurales debido a que el empleo agropecuario se caracteriza por ser independiente o familiar, por este motivo el jefe de hogar (generalmente hombre) es la persona que comúnmente declara ingresos laborales, los que pueden incluir el trabajo de otros miembros del hogar o familia, especialmente de las mujeres.

El Gráfico 7 presenta el cálculo de la brecha mediante la Descomposición de Ñopo incluyendo el área rural. La inclusión del área rural tiene un impacto significativo en el incremento de la brecha de ingresos no explicada, aunque en una menor proporción que en el método Blinder-Oaxaca, el indicador pasa de $21 \%$ a $26 \%$ en 1999; de $16 \%$ a $17 \%$ el 2006, de $11 \%$ a $13 \%$ en el 2013 y en 2018, la brecha de ingreso presenta un ligero incremento desde 5\% hasta $6 \%$.

El Gráfico 8 presenta el impacto en el cálculo de la brecha de ingresos laborales incluyendo la variable ocupación en la Descomposición de Ñopo. La variable fue omitida por su impacto en el porcentaje de hombres y mujeres en el soporte común. Los resultados nos muestran que a pesar de un incremento importante (13pp) en la brecha no explicada para 1999, la brecha no explicada no es estadísticamente diferente del cálculo cuando se omite la variable. El problema central de incluir la variable es la pérdida de validez externa, ya que el soporte común se reduce en 20 pp para las mujeres y $15 \mathrm{pp}$ para los hombres.

15 La encuesta de hogares de 1993 contiene únicamente datos urbanos. 


\section{GRAFICO 6}

\section{BRECHA DE INGRESOS LABORALES NO EXPLICADA UTILIZANDO DESCOMPOSICION BLINDER-OAXACA INCLUYENDO EL AREA RURAL}

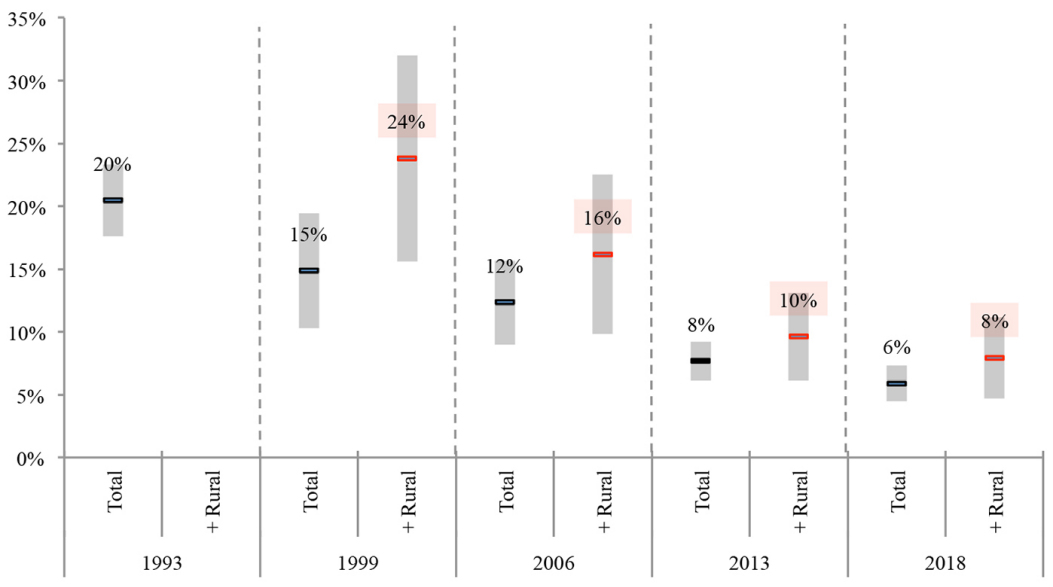

Fuente: Elaboración propia utilizando datos de encuestas de hogares de 1993, 1999, 2006, 2013 y 2018. Las barras presentan el componente no explicado al $95 \%$ de nivel de confianza. La encuesta de 1993 no contiene información del área rural.

\section{GRAFICO 7}

\section{BRECHA DE INGRESOS NO EXPLICADA UTILIZANDO LA DESCOMPOSICION DE NOPO INCLUYENDO EL AREA RURAL}

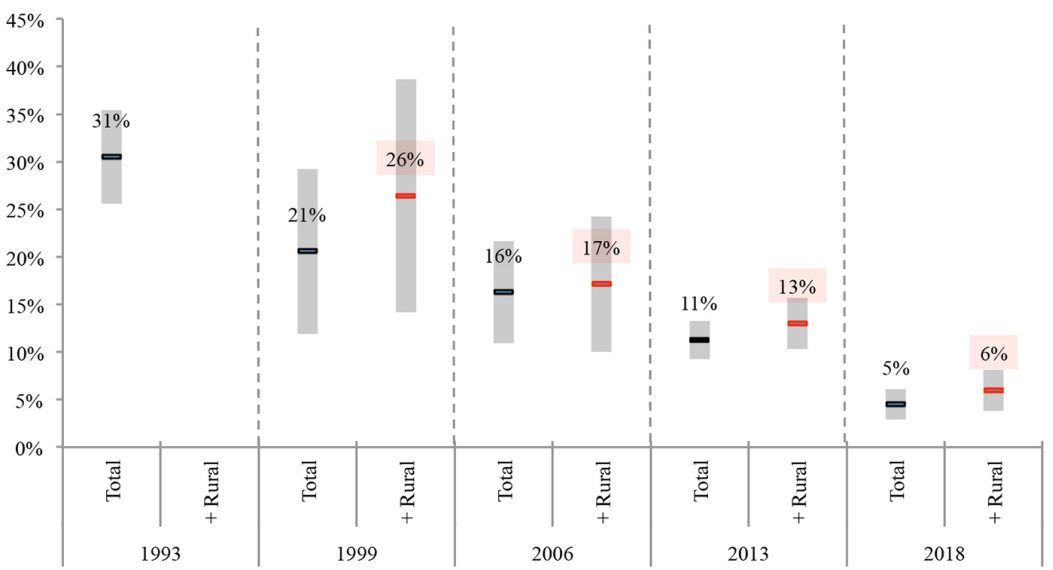

Fuente: Elaboración propia utilizando datos de encuestas de hogares de 1993, 1999, 2006, 2013 y 2018. Las barras presentan el componente no explicado al 95\% de nivel de confianza. La encuesta de 1993 no contiene información del área rural. 


\section{GRAFICO 8}

BRECHA DE INGRESO NO EXPLICADA INCLUYENDO LA VARIABLE OCUPACION UTILIZANDO LA DESCOMPOSICION ÑOPO

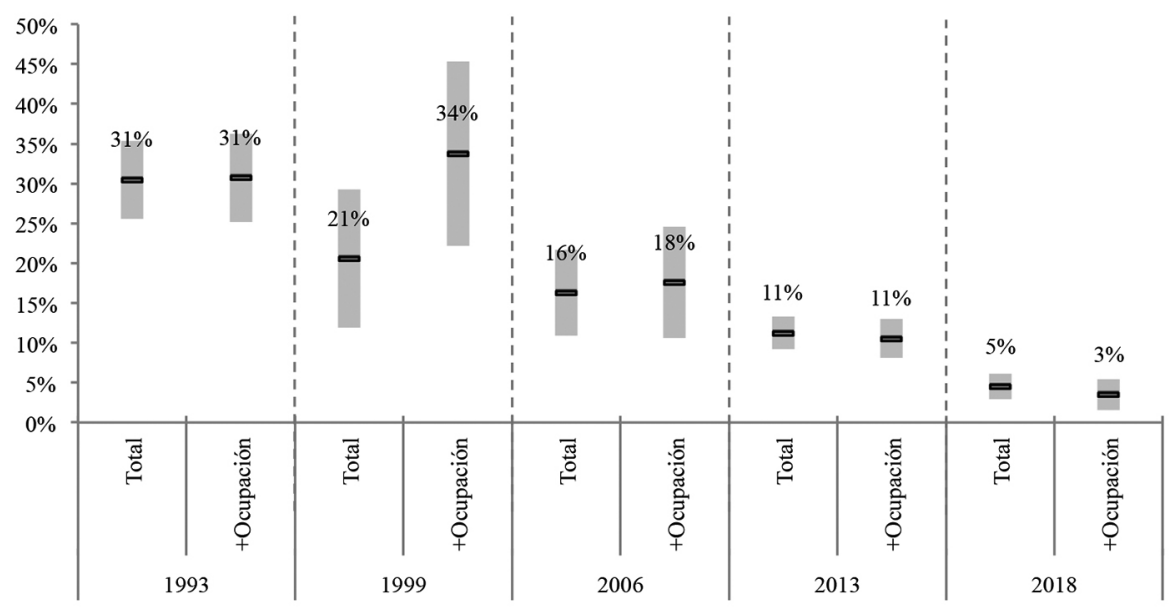

Fuente: Elaboración propia utilizando datos de encuestas de hogares de 1993, 1999, 2006, 2013 y 2018. Las barras presentan el componente no explicado al $95 \%$ de nivel de confianza.

\section{CONCLUSIONES}

En Bolivia las mujeres no solo tienen menores oportunidades en algunos sectores y ocupaciones, sino que reciben menores ingresos que los hombres y se concentran en trabajos con menores salarios. El presente documento tiene como objetivo principal cuantificar y analizar la evolución de la brecha de ingreso por género y proponer explicaciones racionales al comportamiento de la brecha en Bolivia en los últimos 25 años.

Utilizando cinco encuestas de hogares y dos métodos de descomposición del ingreso diferentes: uno paramétrico, la Descomposición Blinder-Oaxaca y otro no paramétrico, la Descomposición Ñopo, encontramos evidencia robusta de una importante reducción en la brecha de ingresos por género utilizando ambos procedimientos, donde destaca que ambos procedimientos muestran una evolución y estimaciones similares. La Descomposición Blinder-Oaxaca nos muestra una reducción de 32\% de la brecha de ingresos en 1993 a 7\% el 2018 y la Descomposición Ñopo nos muestra una reducción en la brecha de $43 \%$ a $7 \%$ en el mismo período.

Esta reducción se explica por la disminución de ambos componentes, el primero explicable por la baja en las diferencias en las características observables, especialmente el relacionada a mejoras en la educación de las mujeres y en el impacto de características 
familiares. El segundo componente que explica la reducción de la brecha se debe a la reducción del componente no explicado, asumido como discriminación. El componente explicado muestra una reducción de $11 \%$ a $1 \%$ mediante la metodología Blinder-Oaxaca y de $8 \%$ a $2 \%$ utilizando el método de Ñopo. El componente no explicado muestra también una reducción de 14 pp o 26 pp, utilizando procedimientos paramétricos y no paramétricos, respectivamente.

Aprovechando la descomposición de Ñopo, se puede explorar las diferenciales de ingreso a base de las características observables, encontramos que la brecha no explicada de ingresos por género se reduce drásticamente para aquellas mujeres con educación terciaria, siendo tan solo 2\% del ingreso promedio de hombres en 2018.

Asimismo, considerando la formalidad, encontramos una ausencia de brecha no explicada por género en el sector formal, mientras que en el sector informal es de casi $7 \%$ del ingreso promedio. Más aún, la brecha total de ingresos por género es negativa para el sector formal y esto se puede atribuir a que las mujeres necesitan una mayor cantidad de credenciales educativas para acceder a mejores trabajos y consecuentemente a mejores ingresos.

Los resultados son robustos luego de la inclusión del área rural. Lo que refuerza los hallazgos e hipótesis acerca de la reducción de la brecha explicada y no explicada (discriminación) en los últimos 25 años.

Estos resultados tienen implicaciones importantes en cuanto a la posibilidad de implementar políticas públicas. Es necesario continuar con la reducción de la brecha de ingresos, para eso es necesario impulsar un acceso igualitario de las mujeres a sectores y ocupaciones que generan mayores retribuciones. Esto se puede alcanzar mediante la capacitación y la inserción laboral de mujeres en ocupaciones y sectores no tradicionales, que les permita mejorar su productividad laboral y consecuentemente sus ingresos laborales. Esto es especialmente relevante para aquellas mujeres con menores niveles de educación y que trabajan en el sector informal. Sin embargo, este tipo de políticas deben estar diseñadas para ser amigables con el rol que ejerce la mujer dentro del hogar y en la sociedad. De esta forma se puede asegurar no solo mejoras en la empleabilidad y en la remuneración, sino también en el bienestar familiar.

\section{REFERENCIAS}

ANDERSEN, L. (1999). "Wage Differentials Between Bolivian Cities". Instituto de Investigaciones Socioeconómicas. Universidad Católica Boliviana.

ANDERSEN, L. (2001). "Low Social Mobility in Bolivia: Causes and Consequences for Development". Instituto de Investigaciones Socioeconómicas. Universidad Católica Boliviana.

ANDERSEN, L., MERCADO, A. y MURIEL, B. (2003). "Discriminación étnica en Bolivia: En el sistema educativo y el mercado de trabajo". Documento de Trabajo, Instituto de Investigaciones Socioeconómicas, Universidad Católica Boliviana, No. 03/03.

ATAL, J., ÑPO H. y WINDER N. (2009). "New Century, Old Disparities: Gender and Ethnic Wage Gaps in Latin America." Inter-American Development Bank, Research Department, Washington, DC. 
BELLONY, A., HOYOS, A. y NOPO, H. (2010). "Gender Earnings Gaps in the Caribbean: Evidence from Barbados and Jamaica". IDB Working Paper Series No. IDB-WP-210.

CEPAL (1992). Bolivia, Encuesta Integrada de Hogares noviembre 1992.

CEPAL (1992). Bolivia, Encuesta Integrada de Hogares noviembre 1994.

CHIODA, L. (2011). "Work and Family: Latin American and Caribbean Women in Search of a New Balance", World Bank. Washington, DC.

CONTRERAS, D. y GALVAN (2003). “HHa Disminuido la Discriminación Salarial por Género y Etnia en Bolivia? Evidencia del Período 1994 - 1999”. IV Reunión sobre Pobreza y Distribución del Ingreso.

CONTRERAS, D. y PLAZA, G. (2010). "Cultural Factors in Women's Labor Force Participation in Chile". Feminist Economics, 16: 2, 27 - 46.

ENAMORADO, T., IZAGUIRRE, C. y ÑOPO, H. (2009). "Gender Wage Gaps in Central American Countries Evidence from a Non-Parametric Approach". IDB Working Paper Series No. IDB-WP-111.

FIELDS, G., LEARY, J., LÓPEZ-CALVA, L. y PÉREZ-DE-RADA, E. “Education's Crucial Role in Explaining Labor Income Inequality in Urban Bolivia". Development Discussion Paper No. 658. October 1998.

GASPARINI, L. y MARCHIONNI, M. (2015). "Bridging Gender Gaps? The Rise and Deceleration of Female Labor Force Participation in Latin America. An overview". Centro de Estudios Distributivo, Laborales y Sociales. Universidad Nacional de La Plata.

HOYOS, A., ÑOPO H. y PEÑA, X. (2010). "The Persistent Gender Earnings Gap in Colombia, 19942006". IDB Working Paper Series No. IDB-WP-174.

JANN, Ben (2008). "The Blinder-Oaxaca decomposition for linear regression models". The Stata Journal (2008). Number 4. pp. 453-479.

NANNESON, F. (2011). "Gender and Income in Bolivia: Using Matching to Decompose Income". Working Paper. University of Copenhagen, Department of Economics.

ÑOPO, H. (2004). "Matching as a tool to decompose wage gaps", IZA Discussion paper series, No. 981.

ÑOPO, H. y HOYOS, A. (2010). "Evolution of Gender Wage Gaps in Latin America at the Turn of the Twentieth Century: An Addendum to New Century, Old Disparities". IZA Discussion Paper Series No. 5086.

ÑOPO, H. (2012). "New century, Old Disparities: gender and ethnic earnings gaps in Latin America and the Caribbean". Inter-American Development Bank. Washington, DC.

NOVELLA, R., RUCCI, G. y VAZQUEZ, C. (2015). "Porque No Quiero o Porque No Puedo ¿Cuánto No Sabemos del Trabajo Femenino en Chile?". IDB technical note 842.

ORGANIZACIÓN INTERNACIONAL DEL TRABAJO (2019). "Panorama Laboral Temático 5: Mujeres en el mundo del trabajo. Retos pendientes hacia una efectiva equidad en América Latina y el Caribe. Lima: OIT / Oficina Regional para América Latina y el Caribe".

OAXACA, R. (1973). "Male-Female Wage Differentials in Urban Labor Markets". International Economic Review, Volume 14, Issue 3.

PSACHAROPOULOS, G. y ZAFIRIS T. (1992). "Latin American Women's Earnings and Participation in the Labor Force." World Bank Policy Research Working Paper 856. Washington, DC, United States: World Bank.

RIVERO, R. y JIMENEZ, W. (1999). "Diferencias saláriales en el mercado de trabajo urbano en Bolivia, 1981-1997". Revista de Análisis Económico, volumen 17, UDAPE.

URQUIDI, M.; VALENCIA, H.; FORONDA, C. CHUMACERO, M. \& BAGOLLE, A. (2018). "Encuesta de Mercado Laboral en Bolivia". Nota Técnica IDB-TN-1450. Banco Interamericano de Desarrollo.

VILLEGAS, H. y NÚÑEZ, J. (2005). "Discriminación étnica en Bolivia: Examinando diferencias regionales y por nicho de calificación”. Documento de Trabajo. Instituto de Investigaciones Socioeconómicas, Universidad Católica Boliviana, No. 02/05.

World Economic Forum (WEF). "Índice Global de Brechas de Genero del Foro Económico Mundial" (2020). 


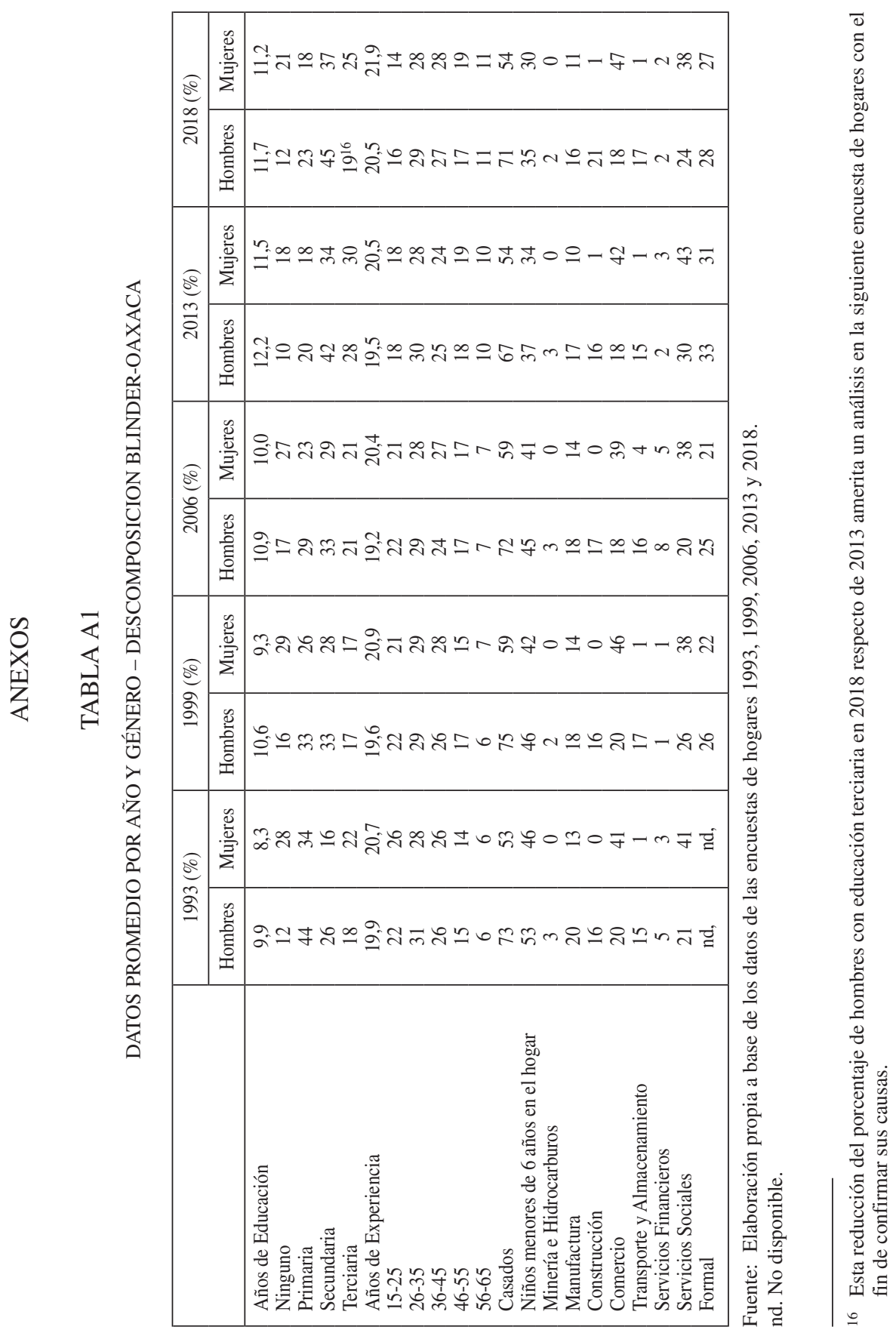


TABLA A2

COMPONENTES DE LA DIFERENCIA EXPLICADA INCLUYENDO OCUPACION BLINDER-OAXACA

\begin{tabular}{|l|c|c|c|c|c|}
\hline & 1993 & 1999 & 2006 & 2013 & 2018 \\
\hline Diferencia Explicada & $0,231 * * *$ & $0,144 * * *$ & $0,117 * * *$ & $0,101 * * *$ & $0,048 * * *$ \\
Educación & $0,092 * * *$ & $0,079 * * *$ & $0,033 * *$ & $0,015 * *$ & 0,001 \\
Experiencia & $-0,004$ & $-0,020$ & $-0,011$ & $-0,004$ & 0,000 \\
Características personales & $0,016 * *$ & 0,010 & $0,017 * * *$ & $0,014 * * *$ & 0,004 \\
y familiares & $0,048 * * *$ & 0,031 & 0,008 & $0,017 *$ & $0,027 * *$ \\
Sector de Actividad & $0,070^{* * *}$ & 0,040 & $0,056 * *$ & $0,055^{* * *}$ & 0,012 \\
Ocupación & $0,009 *$ & $-0,001$ & 0,002 & $-0,001$ & 0,000 \\
Región & n.d. & 0,004 & $0,012 * *$ & $0,005 * *$ & 0,004 \\
Sector Formal & & & & \\
\hline
\end{tabular}

$* p<0.05,{ }^{* *} p<0.01,{ }^{* * *} p<0.001$

Fuente: Elaboración propia a base de los datos de las encuestas de hogares 1993, 1999, 2006, 2013 y 2018.

\section{GRAFICO A1}

BRECHA DE INGRESOS NO EXPLICADA POR FORMALIDAD UTILIZANDO

LA DESCOMPOSICION ÑOPO

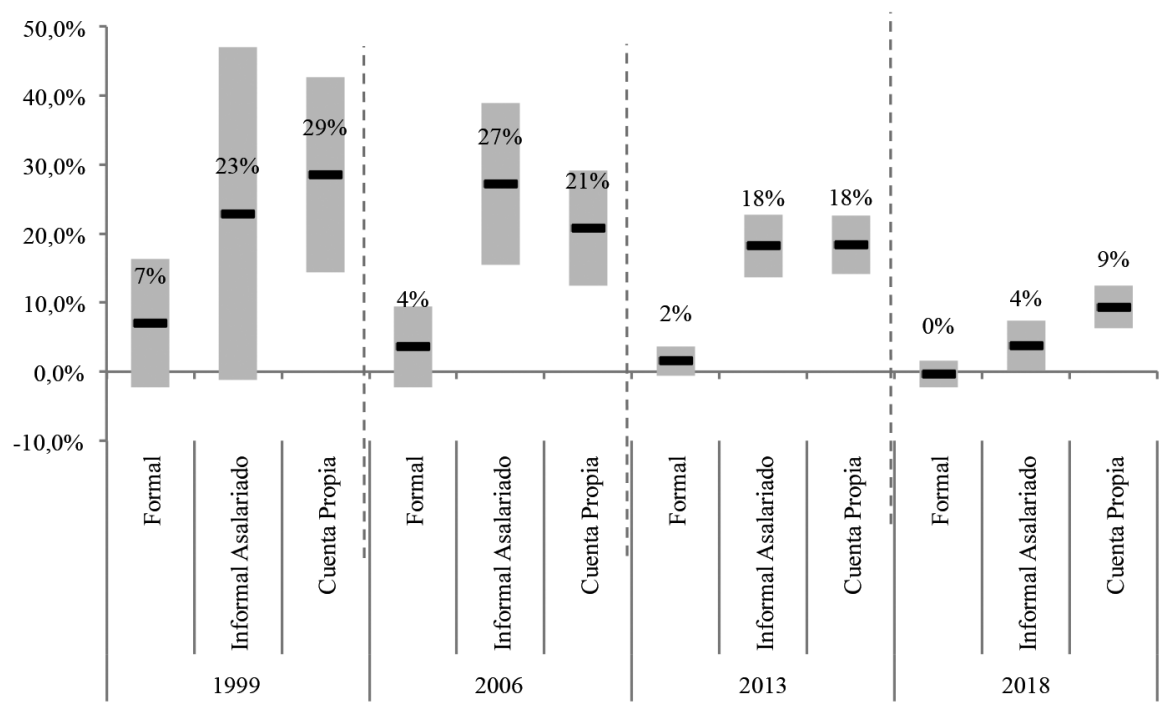




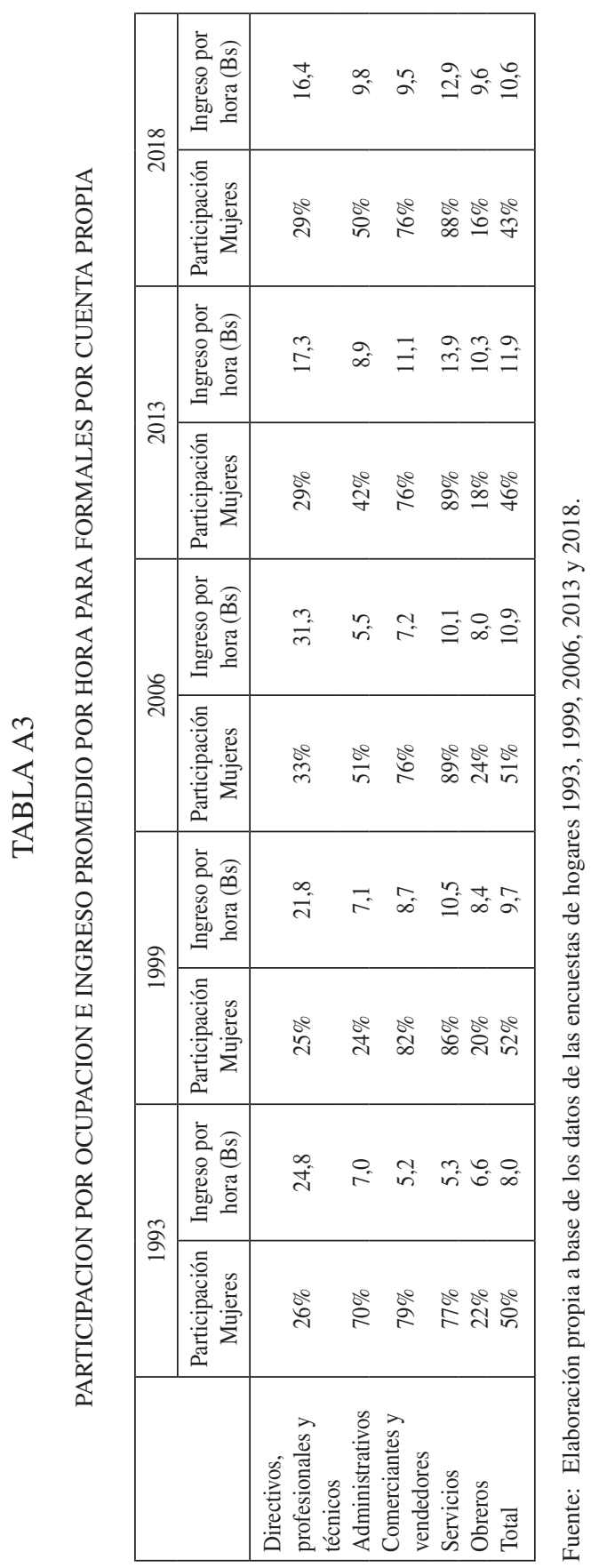




\section{ANEXO 2 \\ DESCOMPOSICION DE FIELDS ${ }^{17}$}

Una forma conveniente y consistente de descomponer la inequidad de ingresos ha sido la desarrollada por Gary S. Fields (Andersen, 1999). Este método se aplica a ecuaciones de ingreso Mincer estándar y requiere multiplicar cada coeficiente de la estimación por la desviación estándar de la variable explicativa y la correlación entre la variable y la variable dependiente, como resultado se obtiene el Ponderador Relativo de Inequidad (F.I.W. por sus siglas en inglés) que se encuentra en una escala que suma 1 (Andersen, 1999).

Formalmente el F.I.W. se calcula de la siguiente manera:

Sea una ecuación Mincer estándar:

$$
Y=\sum_{j} a_{j}^{*} X_{j}
$$

Donde $\mathrm{Y}$ es un vector que contiene el logartimo del ingreso por hora de todos los individuos y $\mathrm{X}$ es la matriz con $\mathrm{j}$ variables explicativas.

Considerando que una medida simple de inequidad es la varianza del logaritmo del ingreso laboral por hora. En este sentido se puede aplicar varianza a ambos lados de la ecuación siguiendo el teorema de Mood, Graybill y Boes que señala que:

Teorema de Mood, Graybill \& Boes: Sea $X_{1}, \ldots, X_{j} y Y_{l}, \ldots, Y_{M}$ dos sets de variables aleatoria y $a_{1}, \ldots, a_{j} y b_{1}, \ldots b_{M}$ dos sets de contantes. Entonces

$$
\operatorname{cov}\left[\sum_{j=1}^{J} a_{j} X_{j} ; \sum_{m=1}^{M} b_{m} Y_{m}\right]=\sum_{j=1}^{J} \sum_{m=1}^{M} a_{j} b_{m} \operatorname{cov}\left[X_{j} ; Y_{m}\right]
$$

$[M G \& B]$

Entonces aplicando el teorema a la ecuación A1 obtenemos:

$$
\operatorname{cov}\left[\sum_{j=1}^{J} a_{j} X_{j} ; Y\right]=\sum_{j=1}^{J} \operatorname{cov}\left[a_{j} X ; Y\right]
$$

Donde el coeficiente de la izquierda es el valor de la covarianza entre Y y ella misma, que es la varianza de Y. Entonces dividiendo ambos lados por la varianza de $\mathrm{Y}$, tenemos

7 Texto adaptado de Andersen (1999) "Wage Differentials Between Bolivian Cities". 


$$
1=\frac{\sum_{j=1}^{J} \operatorname{cov}\left[a_{j} X ; Y\right]}{\sigma^{2}(Y)} \equiv \sum_{j=1}^{J} s_{j}
$$

Donde cada ponderador $\mathrm{s}_{\mathrm{j}}$ está definido como

$$
s_{j}=\frac{\operatorname{cov}\left[a_{j} X_{j} ; Y\right]}{\sigma^{2}(Y)}=\frac{a_{j} \sigma\left(X_{j}\right) * \operatorname{corr}\left[X_{j} ; Y\right]}{\sigma(Y)}
$$

Donde sj son los ponderadores de inequidad que suman a 1 sobre todas las variables explicativas. Cada sj es afectado de forma positiva ante incrementos en el coeficiente $\left(a_{j}\right)$, por la desviación estándar $\left(\sigma\left(X_{j}\right)\right)$ y por la correlación entre la variable explicativa y la variable dependiente $\operatorname{corr}\left[X_{j} ; Y\right]$.

Este método ha sido aplicado en Bolivia por Fields et al. (1998), Andersen (1999) y Andersen (2001) y concluyen que aproximadamente la mitad de la inequidad en el ingreso explicada se debe a la variable educación y las otras variables independientes solo pueden explicar una porción menor de la diferencia en ingresos. Sin embargo, el poder explicativo de las ecuaciones de ingreso es generalmente bajo.

Siguiendo la metodología arriba descrita, nuestras estimaciones para los años estudiados nos demuestran también que entre 1993 y 2018 la educación (tanto en años como en variables dummy de nivel) explicaba aproximadamente la mitad de las diferencias "explicadas" en el ingreso y las otras variables afectan de manera menor la diferencia explicada.

Es interesante notar la disminución en la importancia de la educación como factor de inequidad en el ingreso, posiblemente causado por el incremento del nivel de educación de la población boliviana y el cierre de la brecha de educación. 


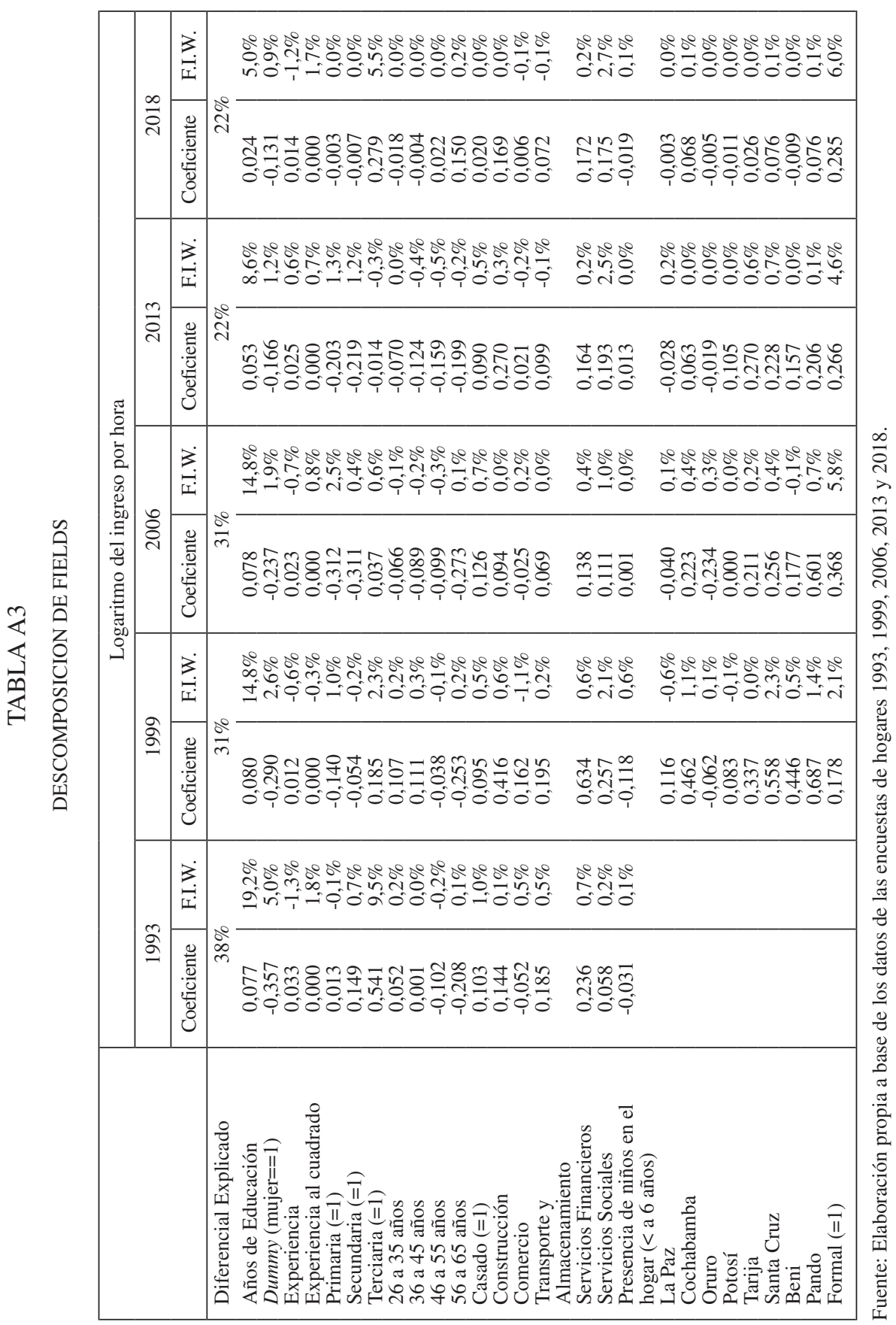

